\title{
Spontaneous adverse drug reaction reporting by patients in Canada: a multi-method study-study protocol
}

Rania Al Dweik ${ }^{1 *}$, Sanni Yaya ${ }^{2}$, Dawn Stacey ${ }^{3}$ and Dafna Kohen ${ }^{4}$

\begin{abstract}
Background: Monitoring adverse drug reactions (ADRs) through pharmacovigilance are vital to patient safety. Spontaneous ADR reporting is one method of pharmacovigilance, and in Canada all reporter types admitted to report an ADR to the Canadian Vigilance Program at Health Canada. Reports are submitted to Health Canada by post, telephone, or via the internet. The Canada Vigilance Program electronically records submitted information to detect medication safety alerts. Although previous studies have shown differences between patients and healthcare professionals (HCPs) on the types of drugs and reactions reported, relatively little is known about the importance of patient reports to pharmacovigilance activities. This article proposed a multi-method approach to evaluate the importance of patient ADR reporting on pharmacovigilance activities, by systematically review the available literature, comparing patient-versus HCPs-generated ADR reports that were submitted to the Canada Vigilance Program, and exploring patient views and experiences regarding the Canadian ADR reporting system.
\end{abstract}

Methods: Guided by a risk-perception theoretical lens, the proposed multi-methods research study will involve three phases. Phase I is a systematic review of all studies that analyse the factors influence ADR reporting by patients to the pharmacovigilance schemes. Phase II is a descriptive statistical analysis of all ADR reports received by the Canada Vigilance Program database between 1 January 2000 and 31 December 2014 from patients and HCPs to compare ADRs reported by patients with those reported by HCP reports in terms of ADR seriousness, ADR classification by system organ class, and the medication involved based on the anatomical therapeutic class system. In phase III, an interpretative descriptive approach will be used to explore patient's views and experiences on ADR reporting and usability of the Canadian Vigilance ADR report. Participants will be purposefully selected to ensure diverse backgrounds and experiences. Interviews will be digitally-recorded, transcribed verbatim, and inductively analysed to identify themes. Patients will be interviewed until theoretical saturation is achieved.

Discussion: Findings from this research will highlight the role of the patients in directly reporting ADRs, and provide information that may guide streamline and optimizing patient ADR reporting. Policy makers, public health officials, and regulatory agencies will require this critical information in order to improve medication safety in Canada and worldwide.

Keywords: Adverse drug reactions (ADRS), Pharmacovigilance, Patient reporting, Canada

\section{Background}

Adverse drug reactions (ADRs) are a worldwide problem that affects all drugs and their users. They cause

\footnotetext{
*Correspondence: raldw034@gmail.com

1 Population Health Program, Faculty of Health Science, University of Ottawa, Ottawa, ON, Canada

Full list of author information is available at the end of the article
}

significant disability and mortality, and are expected to be associated with an economic drain on the healthcare system (Bates et al. 1995; Oshikoya and Awobusuyi 2009). ADRs are monitored in many countries and by the World Health Organization (WHO) since the 1960s using spontaneous reporting systems, also called 'early warning' systems (Stricker 2004). Pharmacovigilance is the science

\section{垈 Springer}


and activities relating to monitoring, detection, assessment, understanding, and prevention of ADRs (Aagaard et al. 2009). Monitoring product safety has been traditionally done by passive surveillance (voluntary reports) or the collection of spontaneously reported adverse events from healthcare providers and consumers following the administration of a medicinal product (Sharrar and Dieck 2013).

In the beginning of ADR monitoring, only doctors and dentists were allowed to submit ADR reports to these databases (Aagaard et al. 2009). But, because the health agencies started focusing more on patients' safety, in 1995 all drug manufacturers, world widely, were mandated to report ADRs (WHO 2002). Later, other healthcare professionals (HCPs), pharmacists, and patients were allowed to report ADRs in hope that this would increase the volume and quality of ADR reports (Aagaard et al. 2009).

Spontaneous direct patient reporting may prove to be essential for continuous improvement and successful pharmacovigilance. However, the literature is deficient regarding the role of direct patient reporting, and its effects, on pharmacovigilance activities (Herxheimer et al. 2010). Much controversy remains among experts concerning the utility and efficacy of incorporating patient ADR reports into pharmacovigilance guidelines and protocols (Mitchell et al. 1988; Hazell et al. 2013a, b). Some researchers believe that patient ADR reporting is detrimental to pharmacovigilance activities while others fully support it and agreed that direct patient reporting is necessary to add that extra layer for good pharmacovigilance since patients as users of medications have first-hand knowledge of their experiences with ADRs (Aagaard et al. 2009; van Grootheest and de, Graaf L, de, 2003; Blenkinsopp et al. 2007).

To date, few countries, including Canada, recognize patient-submitted ADR reports in their pharmacovigilance activities (Aagaard and Hansen 2010). One of the studies showed that the quality of direct patient reporting has been found to be similar to that of reports from HCPs and the spontaneous patients reporting of serious adverse drug reactions were helpful in detecting drug alert (Aagaard et al. 2009; Blenkinsopp et al. 2007). Other study showed that compared with other sources, patients reported different categories of ADRs for different types of medicines (Aagaard et al. 2009). Patients, through their reports, can offer a more direct and precise view of what is actually occurring during the treatment period (Blenkinsopp et al. 2007). The reports from patients provide insight that reports from HCPs simply cannot. Unlike reports from physicians, these reports describe how the adverse effects affect people's lives (Herxheimer et al. 2010). Patient ADRs have the potential to express descriptive details of the ADR.

Patients often differ in their risk perception with respect to drug therapy when compared to HCPs (Aronson 2006). In one of the studies that conducted in the UK, patients' reports differ from HCPs reports in terms of categorising event types (Blenkinsopp et al. 2007). In another study participants were requested to provide their risk perception of ADRs associated with 13 different drug classes. The findings showed that the two groups perceived ADR risks differently. Among HCPs, anticoagulants and non-steroidal anti-inflammatory drugs [NSAIDs] were ranked as the most dangerous drugs with respect to ADRs, whereas patients did not rank NSAIDS among the most dangerous drugs and believed that aspirin [an NSAID] posed the least threat (Bongard et al. 2002). In Denmark, consumers were more likely to report ADRs from SOCs: 'nervous system disorders'; 'psychiatric disorders' and 'reproductive system and breast disorders' (than other sources (Aagaard and Hansen 2010).

Furthermore, by allowing direct patients spontaneous reporting, we are allowing the patient to be actively involved in their treatment. With the added patient reports to those of HCPs, the spontaneous surveillance database can acquire data rich in additional information about ADRs (Hammond et al. 2007). It could be argued that this only speeds up the drug alert detection process.

The literature review has demonstrated a gap in knowledge of one of the earliest safety reporting system active since 1965. No studies were found that compare and contrast patient reports against $\mathrm{HCP}$ reports in Canada using adverse reaction reporting system of Canada. Understanding how patients are involved in ADR reporting can provide new insights into the importance and limitations of integrating patient-generated ADR reports into pharmacovigilance approaches which might help in improving medication use safety in Canada. This study aimed to provide new insights into the importance of patients ADR reporting. Policy makers, public health officials, and regulatory agencies require this critical information to improve medication safety.

\section{Objective and research questions}

The objective of this protocol is to evaluate the importance of patient ADR reporting on pharmacovigilance activities, by systematically review the available literature, comparing patient-versus HCPs-generated ADR reports-based on seriousness, system organ class-anatomical therapeutic class-that were submitted to the Canada Vigilance Program, and exploring patient views and experiences regarding the Canadian ADR reporting system. The study will involve three phases that will focus on the following research questions: 
1. What are the factors influencing reporting of adverse drug reaction by patients?

2. What types of ADRs, suspect drug classes, reaction severity, and ADR-involved organ systems are reported by patients versus HCPs?

3. What are the views and experiences of patients regarding ADR reporting and the user-friendliness of ADR reporting in Canada?

\section{Conceptual framework}

The Risk Perception Theory will provide a theoretical lens to examine the differences between patients and HCPs perceptions in term of the number of serious ADRs reported by each group. It works on the concept that different classes of people [professionals versus nonprofessionals, scientists versus the public, or, in this case, HCPs versus patients] have different views on the possible risks associated with some action or environment (Af WÅhlberg AE. 2001).

\section{Methods}

This study protocol consists of three complementary phases, each phase sequentially informs the next.

\section{Phase I: Systematic review of all studies that analyse the factors influencing reporting ADRs by patients to the pharmacovigilance systems}

Research question 1 What are the factors influencing reporting of adverse drug reaction by patients?

\section{Methods}

A systematic literature review will be guided by the Cochrane Handbook (Higgins and Green 2011), and will be structured to meet the Preferred Reporting Items for Systematic Reviews and Meta-Analyses (PRISMA) (Moher et al. 2009) criteria. Eligible studies addressed the factors influencing ADR reporting by patients. The search strategy protocol will be developed collaboratively with an academic reference librarian to refine queries and characterize them in terms of elements. Study inclusion/exclusion criteria will be framed in a systematic manner, using the elements of a question and include population, intervention, comparator, and outcome (PICO) (Stillwell et al. 2010) (Table 1) even if all these elements are not used in the formal search strategy.

\section{Search strategy}

The search will focus on all major databases relevant to the subject matter, i.e., PubMed, MEDLINE, EMBASE, CINAHL, PSYCINFO, ProQuest Dissertation and Theses, and Cochrane library. These databases will be searched using both medical subject heading (MeSH) and text search terms including: patients, consumers, public, adverse drug reactions, report, reporting, spontaneous, pharmacovigilance, and surveillance. The search dates will include all years for the database, i.e., from the earliest data sources on each database, e.g., 1947 or earlier, and up to December 2014. The reference lists of each included study will be checked to identify additional studies for potential inclusion. Grey literature searches will be performed using similar search terms.

\section{Data extraction}

All data from the included studies will be extracted using a data extraction form. The form will be pilot tested prior to commencing data extraction. After the identification of studies, duplicates will be removed and two independent reviewers will conduct three levels of screening. Level one of screening is to determine study relevance to the overall objective of the systematic review and this will include title screening. Level two screening will be a title and abstract screen based on the inclusion/exclusion criteria. Level three screening will include reviewing the final group of studies identified by both reviewers as meeting the inclusion criteria. A second reviewer will verify the accuracy of extracted data. Study data quality and study bias risk assessments will be performed. The two independent reviewers will assess the quality of included studies using the appropriate critical appraisal skills programme (CASP) checklist (Milne and Oliver 1996).

Table 1 Systematic review inclusion/exclusion criteria

\begin{tabular}{|c|c|c|}
\hline & Include & Exclude \\
\hline Study Design & $\begin{array}{l}\text { Regardless of methodology, eligible studies answered the } \\
\text { question: what factors influence reporting ADRs by patients? }\end{array}$ & - \\
\hline Population & $\begin{array}{l}\text { Studies addressing patients view and perceptions on ADRs } \\
\text { reporting }\end{array}$ & $\begin{array}{l}\text { Studies addressing: the view and perception of HCPs on ADRs } \\
\text { reporting, the role of HCPs in ADR reporting, ADRs reported by } \\
\text { HCPs, restricted to specific drugs or therapeutic class }\end{array}$ \\
\hline Setting & No restriction on setting & - \\
\hline Language & $\begin{array}{l}\text { All studies in English. Non-English studies will be converted } \\
\text { to English using Google Translate (due to resources limitation) }\end{array}$ & - \\
\hline
\end{tabular}




\section{Data analysis}

The data will be synthesized by classifying the different study types, and by comparing and contrasting study findings. Characteristics of included studies will be analysed descriptively and the results will present in a narrative format recommended by the PRISMA (Preferred Reporting Items for Systematic Reviews and Meta-Analyses) statement (Liberati et al. 2009).

\section{Bias}

A prior plan was made to minimize the risk of bias (Hartling et al. 2009) using Assessment of Multiple Systematic Reviews (AMSTAR) (Shea et al. 2009). The systematic review protocol was established, and the two researchers will be involved in screening the identified literature and will participate in data extraction.

\section{Phase II: Observational retrospective descriptive statistical comparison of ADR reports from patients versus healthcare professionals Research questions and hypothesis}

1. Are there a significant difference in distribution of ADRs between reports (patients vs. HCPs) based on ADRs seriousness)? Does the trend change?

2. Are there significant differences in distribution of ADRs between reporters (patients' vs HCPs) for system organ classification $(\mathrm{SOC})$ ?

3. Are there significant differences in distribution of ADRs between reporters (patients' vs HCPs) for the suspected drug class involved?

\section{Methods}

\section{Study design}

An observational-retrospective cross-sectional study design will be used. The study will include all ADR reports submitted to the Canadian Vigilance Database from January 2000 through December 2014, inclusive. Using 14 years data will help to observe the changes in patients reporting. The ADR report analysis will compare content from patients-versus HCPs-submitted reports in terms of ADR seriousness, ADR classification by system organ class, and the medication involved based on the anatomical therapeutic class system (ATC). Only ADRs classified as 'serious' will be analysed because identifying serious ADRs are the primary focus of spontaneous reporting systems and are of particular public health interest. The unit of analysis will be the ADR.

\section{Study setting}

The Canada Vigilance database contains all spontaneous ADR reports in Canada that were reported directly by HCPs, patients, and pharmaceutical companies. An ADR report is defined by the following four criteria, which must be included in all reports: (1) patient information; (2) the suspected medicine(s); (3) patient outcome information (s); and (4) reporter. Reports are categorized in the database by the degree of seriousness according to the Council for International Organizations of Medical Sciences criteria (Health Canada 2007). Health Canada Vigilance handles the ADR reports from the patients the same way it handles reports from other sources. The Canadian Vigilance database defines the following four categories of persons submitting reports to the database as: (1) community including patients, patients' relatives, other members of the public; (2) hospital including HCPs such as physicians, pharmacists, and nurses; (3) MAHs including pharmaceutical companies; and (4) others including insurance companies.

\section{Data extraction}

Anonymized data will be extracted from the Canadian Vigilance ADR database based on the date reports were received, category of person submitting the reports, reaction seriousness, medications involved, and category of ADRs classified by SOC. The reported ADRs were coded according to the Medical Dictionary for Regulatory Activities (MedDRA) structure on SOC. The Canadian Vigilance database ADR search criteria include either the trade names or generic name of medications reported to cause ADRs. Therefore, it will be necessary to manually translate the trade names and generic names in the pharmacological/therapeutic subgroup of ATC level 1 to present the ADR data in a comprehensive format (Aagaard et al. 2009).

\section{Medical dictionary for regulatory activities (MedDRA)}

The Medical Dictionary for Regulatory Activities (MedDRA) was developed by the International Conference on Harmonization (Journot et al. 2008). It is clinically validated international medical terminology used by regulatory authorities and the regulated biopharmaceutical and pharmaceutical industries (MedDRA 2009). MedDRA is needed for electronic exchange of information on adverse reactions between industry and regulatory authorities after drug registration in the European Union (Brown 2004). The terms in MedDRA are numerically coded to support electronic means of data transmittal and retrieval. It has 26 broad groups called system organ classes. MedDRA was used for coding ADR reported by HCPs and patients in this study. The SOC will be identified via this method and compared according to reporter.

\section{Anatomical therapeutic chemical system (ATC)}

The Anatomical Therapeutic Chemical (ATC) system classifies medicinal products according to their primary constituent, the organ or system on which they act, and 
their chemical, pharmacological and therapeutic properties (Aagaard et al. 2009). Medicinal products are classified into groups on five different levels. The medicines are divided into 14 main groups (first level), with one pharmacological/therapeutic subgroup (second level). The third and fourth levels are chemical/pharmacological/ therapeutic subgroups, and the fifth level is the chemical substance. The second, third, and fourth levels are often used to identify pharmacological subgroups if this is considered more appropriate than the therapeutic or chemical subgroups (Methodology WCCfDS 2011). In this study, ATC level one will be used to comprehensively categorize ADR data into one of 14 groups.

\section{Data analysis}

The main goal of the statistical analysis is to investigate the dependence between the reporter and the reported ADR (classified by seriousness, SOC, and ATC) using the "null hypothesis". It will be assumed that there are no differences in the number of serious ADRs reported by patients versus HCPs. In case the null hypothesis is rejected and to ensure that any reporting differences between patients and HCPs will be indeed significant and not due to chance, the data will be analysed using the Chi-squared test for independence. This test is used to determine whether the frequency of each group is different from what would be expected. If the calculated Chi-square is high enough, then the frequencies found would not be expected on the basis of chance and the null hypothesis will be rejected. Odds ratios (ORs) will be calculated using a $2 \times 2$ table for the reporter category (patients and HCPs), SOC, or ATC. Confidence intervals will be calculated for all ORs (95\% level). Statistical analyses will be performed using SPSS version 17 statistical software.

\section{PHASE III: Explore patient views and experiences} on reporting ADRs to the Canadian vigilance database Research question 3 What are the factors influencing patient ADR reporting? What are the views and experiences of patients regarding the user-friendliness of ADR reporting in Canada?

\section{Method}

\section{Study design}

An interpretive descriptive qualitative study will be conducted. Interpretive description is an inductive and effective analytic approach for describing health care events (Thorne et al. 2009).

\section{Participants and setting}

Eligible participants will be adult patients over 18 years old living in Canada, have experienced an ADR, and able to answer questions in English or French. Purposeful sampling will be used to represent a range of ages (18-45, 45-64, 65-80), sex (male, female), and patients who have reported a side effect to pharmacists, physicians, nurses, or to the Canada Vigilance Database. If we encounter difficulty-finding participants, we will revert to the snowball technique. This approach is a sampling strategy that consists of seeking referrals from participants, where one participant gives the researcher the name of more possible participants (Creswell 1998). This technique is used in populations where it is difficult to identify potential participants. Recruitment will continue until data saturation plus an additional three participants, to ensure that no new themes will be identified (Francis et al. 2010). We anticipate reaching saturation with 10 participants and therefore recruiting a total of 13 patients.

Participant recruitment Patients will be informed about study details and invited to participate through social media (e.g. Kijiii, Facebook, twitter), patient associations, and patient societies such as Patients Canada, Canadian Arthritis Society, or Canadian Cancer Society or Heart \& Stroke.

\section{Data collection}

First, an email or pamphlet introducing the study will be given to prospective participants that express an interest in the study. Those willing to participate will have an interview set up at a convenient time and location (including in person, telephone, or skype). During the interview, the researcher will explain the purpose of the study, describe the approximate amount of time it will take to complete the interview, and inform the participant that they can withdraw from the interview at any time or refuse to answer any questions. Participants will also be informed of precisely how their data will be used, and an informed consent form will be provided for approval, signature, and return. The interview will be conducted using a semi-structured interview guide and is expected to take $30-45 \mathrm{~min}$. The semi-structured interview guide was developed based on key sensitizing concepts from the literature on ADR reporting and with review by experts in pharmacy, knowledge translation to patients, and health policy. More specifically, the first set of questions will explore participant experiences with medication-related side effects, severity, who they report side effects to, and expectations about reporting. The second set of questions will focus on the userfriendliness of the ADR reporting form that is available on the Canada Vigilance website. The interviewer will lead the patient through the form step-by-step and then ask questions exploring the clarity of the questions, ease of use, readability, and font size. Non-identifying demographic information will also be collected. Interviews 
will be audio-taped and field notes documented by the researcher.

\section{Data analysis}

Interview audiotapes will be transcribed verbatim and analysed. Participant responses to the semi-structured interview questions will be analysed using interpretive content analysis (Huberman and Miles 1994). Interpretive content analysis is an iterative process that facilitates analysis of text that describes processes, activities, perceptions, and beliefs (Thorne et al. 2009). The qualitative data analysis process will use the three-step method of data reduction, data display, and drawing conclusions/ verification (Huberman and Miles 1994). Several techniques will be used to enhance the credibility, auditability, and transferability of the study findings (Lincoln and Guba 1986).

To enhance credibility, several techniques will be used including: (a) use of open-ended interviewing techniques: tape recording and verbatim transcriptions to increase the accuracy of describing each participant's experience;(b) share interpretation of findings with participants to enhance accuracy from the perspective of the person's living experience; and (c) extensive literature review (Lincoln and Guba 1986).

Auditability is the second assessment of accuracy (Lincoln and Guba 1986). This will be enhanced through presentation of enough information to the reader to see how the raw data lead to the interpretation.

The third criterion of assessment is transferability of the data by presenting enough details on the finding that will allow others in the discipline to determine the relevance of the study finding for their own practice, research, and theory development.

\section{Strengths and limitations}

Using secondary data from the official website of the Health Canada database in Phase II to compare serious ADRs reported by HCPs versus patients is considered cost-effective and time-efficient. Since the database contains a large number of ADR reports; sample size is not a problem. But because there is no limitation on the ATCs in the database, this would result in a broader scope of information that will be obtained and more manual work will be needed. One of the study limitation that needs to be addressed is the fact that one reporter can report more than one ADR per report, thus resulting in ADRs not being truly independent. It is important to note that when analysing data obtained from the Canada Vigilance Database, causality of a reaction by any given suspect drug cannot be assumed. In addition, comparisons between drugs cannot be made from the available data (Health Canada 2011).
Using the interpretive descriptive approach in Phase III will create an opportunity to understand the patient perception toward adverse drug reaction reporting and the Canadian Vigilance system which will help in interpreting and understanding the results from phase II. (Thorne et al. 1997). In phase III, efforts to include participants with various demographic characteristics, an attempt to broaden the recruitment, will be conducted by providing posters to patient associations, and use advertisements and Internet resources to facilitate participation.

\section{Ethic}

Secondary data obtained from the official website of Health Canada will be used in this research. Secondary data analysis does not pose a privacy risk to participants the way that primary data collection and analysis might, but the rights of participants will still need to be protected. All patient data will be anonymized and this study protocol had received approval of the University of Ottawa Research Ethics Boards in July 2014 (H05-14-18).

The ethical aspects of the research involving human participants will follow the guidelines of the national granting councils (the Canadian Institutes of Health Research) set out in the Tri-Council Policy Statement on Ethical Conduct for Research Involving Humans (Ethics PoR. Tri-Council Policy Statement: Ethical Conduct for Research Involving Humans (TCPS) 2001).

For ethics approval, four major ethical concerns were considered: risk, benefit, consent, and confidentiality. The study is not anticipated to involve direct risk to participants because no active intervention will occur. Participants will be aware that participation will be voluntary and that they are free to skip any question(s) that make them uncomfortable, and can withdraw from the study at any time.

Benefit will be explained as an opportunity to inform the scientific community and policy makers on current ADR issues and concerns. After participation, interviewees will be given the opportunity to ask about current reporting procedures to gain knowledge on the subject. There is no financial benefit to study participants. Each participant will provide written informed consent prior to participate in the study. The consent form will be explained prior to beginning the study and questions will be answered. Participants will be informed that steps to ensure confidentiality will be taken. All interviews will be conducted in a one-on-one manner to augment participant confidentiality. Participants will be identified in all written reports by numbers only, not by names. Any identifying information will be removed from the transcripts and all electronic files will be kept password protected, while hardcopy files will be kept in a locked cabinet at researcher student office-Health Science Department-University of Ottawa. 


\section{Discussion}

Adverse drug reaction reporting systems need to be robust in order to be able to detect new drug alerts and improve pharmacovigilance. These systems are necessary to identify new reactions and to prevent potential ADRs. The purpose of this research is to enhance the understanding of safety issues associated with medication use.

The methodology proposed here has several strengths. First, we will systematically review all published studies that involve patients reporting to national agencies and compare that with what has been done in Canada. Second, we will descriptively analysis all ADR reports received by the Canada Vigilance Program database between January 1st 2003 and December 31st 2012 from patients and HCPs and compare these submitter groups in terms of: seriousness of suspected ADRs reported, distribution of ADR reports based on affected organ system(s), and the suspected medicine classes involved. And third, we will use an interpretative descriptive approach to explore patients' views and experiences on ADR reporting and usability of the Canadian Vigilance ADR report. This will create an opportunity to understand patient perceptions toward the Canadian Vigilance system and will help in interpreting and understanding the results from descriptive analysis of the data collected. One of the advantages of using the interpretive descriptive methods is the ability to collect accurate data and provide a clear picture of the description under study (Ethics PoR 2001).

Findings from this research will make three significant contributions: (1) it will highlight the role and process of patients in directly reporting ADRs; (2) give new information that may guide streamline in optimizing patient ADR reporting and; (3) provide policy makers, public health officials, and regulatory agencies with this critical information in order to improve medication safety in Canada.

\section{Abbreviations}

ADR: adverse drug reactions; HCPs: healthcare professionals; ATC: anatomical therapeutic chemical; SOC: system organ classification; CASP: critical appraisal skills programme (Milne and Oliver 1996); AMSTAR: assessment of multiple systematic reviews.

\section{Authors' contributions}

$\mathrm{RD}$ is the primary investigator, conceived, led and co-ordinated the development and writing of the manuscript; SY, DS, and DK, participated throughout the development and writing of the manuscript by contributing intellectual content and feedback on drafts of the manuscript. All authors read and approved the final manuscript.

\section{Author details}

${ }^{1}$ Population Health Program, Faculty of Health Science, University of Ottawa, Ottawa, ON, Canada. ${ }^{2}$ School of International Development and Global Studies, Faculty of Social Science, University of Ottawa, Ottawa, ON, Canada. ${ }^{3}$ Ottawa Hospital Research Institute, School of Nursing, Faculty of Health Sciences, University of Ottawa, Ottawa, ON, Canada. ${ }^{4}$ School of Epidemiology, Public Health, and Preventive Medicine, University of Ottawa, Ottawa, ON, Canada.

\section{Acknowledgements}

I would like to thank Ms. Lindsey Sikora, librarian at University of OttawaHealth Science, for her contributions in preparing phase I of the manuscript.

\section{Competing interests}

The authors declare that they have no competing interests. 


\section{Appendix: Patient interview guide}

Background Information for Patient Interview to be filled out by researcher

\section{Questions for Patients Guided Interview}

1. Tell me about your experiences with side effects from taking medications? (Prompt: who do you tell, when, what was the response, who reported?

2. Do you describe the side effect as "bad" or "serious" ? (tell me what do you mean by bad or serious"?

3. Are you aware of the Canadian Vigilance System for reporting side effects from medications? a.

$\square$ Yes b. $\square$ No

(if yes: please tell me what you know about this system?)

(In case of non-face to face interview, the Canadian adverse drug reaction report form will be sent to the participant). Introduce the form by walking the participants through the six sections of the form (Personal Information about the Reporter, Health information about the Reporter, The medication involved in side effect, Other health products used at the same time, Information about the outcome after side effect, and Information about the outcome after side effects) in two minutes.

4. What were your first impressions about the ADR reporting form?

5. Is there questions missing that you would like to see added or removed from the side effect reporting form?

6. Did you find that the questions included in the side effect reporting form were:

$\square$ Much less than I expected

$\square$ Little less than I expected

$\square$ About right 
$\square$ Little more than I expected

$\square$ Much more than I expected

7. Are the font and icons (check boxes etc.) readable (i.e. font type and size)? $\square$ Yes $\square$ No

8. Is there enough space for data entry? $\square$ Yes $\square$ No

9. For the next questions, I will like to ask you to rate each section of the side effect report by choosing 'poor', 'fair', good', or 'very good' to show what you think about the clarity of the questions:

9.1 Personal Information about the Reporter

9.2 Health information about the Reporter

9.3 The medication involved in side effect

9.4 Other health products used at the same time

9.5 Information about the side effect

9.6 Information about the outcome after side effect

$\square$ poor $\square$ fair $\square$ good $\square$ very good
$\square$ poor $\square$ fair $\square$ good $\square$ very good
$\square$ poor $\square$ fair $\square$ good $\square$ very good
$\square$ poor $\square$ fair $\square$ good $\square$ very good
$\square$ poor $\square$ fair $\square$ good $\square$ very good
$\square$ poor $\square$ fair $\square$ good $\square$ very good

10. Would you use the form or tell someone about the side effect reporting form?

Yes $\square$ No $\square$ Not sure

11. Do you have any further comments on the side effect reporting form? Or your experience with reporting side effects?

12. What is your age range?

Under 30

$\square 30-40$

$\square 41-50$

51- 60

$\square 61-70$

$\square 70$ and older

13. Your sex:

Female $\square$ Male

14. What is the highest level of education you have completed? 
Less than high school

\author{
$\square$ High School
}

Community College

University undergraduate degree

$\square$ University graduate degree

15. What is your occupation?

Thank you for taking the time to go through this interview with me.

Received: 9 May 2015 Accepted: 15 February 2016

Published online: 29 February 2016

\section{References}

Aagaard L, Hansen EH (2010) Consumers'reports of suspected adverse drug reactions volunteered to a consumer magazine. Br J Clin Pharmacol 69(3):317-318

Aagaard L, Nielsen LH, Hansen EH (2009) Consumer reporting of adverse drug reactions: a retrospective analysis of the Danish adverse drug reaction database from 2004 to 2006. Drug Saf 32(11):1067-1074

Af WÅhlberg AE (2001) The theoretical features of some current approaches to risk perception. J Risk Res 4(3):237-250

Aronson J (2006) Risk perception in drug therapy. Br J Clin Pharmacol 62(2):135-137

Bates DW, Cullen DJ, Laird N, Petersen LA, Small SD, Servi D, Laffel G et al (1995) Incidence of adverse drug events and potential adverse drug events. Implications for prevention. ADE Prevention Study Group. JAMA 274(1):29-34

Blenkinsopp A, Wilkie P, Wang M, Routledge PA (2007) Patient reporting of suspected adverse drug reactions: a review of published literature and international experience. Br J Clin Pharmacol 63(2):148-156

Bongard V, Ménard-Taché S, Bagheri H, Kabiri K, Lapeyre-Mestre M, Montastruc $J$ (2002) Perception of the risk of adverse drug reactions: differences between health professionals and non health professionals. Br I Clin Pharmacol 54(4):433-436

Brown EG (2004) Using MedDRA. Drug Saf 27(8):591-602

Creswell J (1998) Qualitative inquiry and research design: choosing among five traditions. Sage, Thousand Oaks

Ethics PoR (2001) Tri-council policy statement: ethical conduct for research involving humans (TCPS) [cited 2014]. http://pre.ethics.gc.ca/eng/index

Francis JJ, Johnston M, Robertson C, Glidewell L, Entwistle V, Eccles MP et al (2010) What is an adequate sample size? Operationalising data saturation for theory-based interview studies. Psychol Health 25(10):1229-1245

Hammond IW, Rich DS, Gibbs TG (2007) Effect of consumer reporting on signal detection: using disproportionality analysis. Expert Opin Drug Saf 6(6):705-712

Hartling L, Ospina M, Liang Y, Dryden DM, Hooton N, Krebs Seida J et al (2009) Risk of bias versus quality assessment of randomised controlled trials: cross sectional study. BMJ 339:b4012
Hazell L, Cornelius V, Hannaford P, Shakir S, Avery AJ (2013a) How do patients contribute to signal detection?: A retrospective analysis of spontaneous reporting of adverse drug reactions in the UK's Yellow Card Scheme. Drug Saf 36(3):199-206. doi:10.1007/s40264-013-0021-2

Hazell L, Cornelius V, Hannaford P, Shakir S, Avery A (2013b) How do patients contribute to signal detection? Drug Saf 36(3):199-206

Health Canada (2007) About MedEffect Canada [cited 2014 Feb 2]. http:// www.hc-sc.gc.ca/dhp-mps/medeff/about-sujet-eng.php-share

Health Canada (2011) Canada Vigilance Program-Collecting and Assessing Adverse Reaction Reports [updated 2011-04-28; cited 2014 Jan, 24]. http://www.hc-sc.gc.ca/dhp-mps/alt_formats/pdf/pubs/medeff/fsif/2011-cvp-pcv/2011-cvp-pcv-eng.pdf

Herxheimer A, Crombag R, Alves TL (2010) Direct patient reporting of adverse drug reactions: a 12-country survey. Briefing Paper. Health Action International, Europe

Higgins JP, Green S (2011) Cochrane handbook for systematic reviews of interventions. Wiley Online Library, New York

Huberman AM, Miles MB (1994) Data management and analysis methods. Sage Publications, Thousand Oaks, CA, US

Journot V, Tabuteau S, Collin F, Molina J-M, Chene G, Rancinan C (2008) About the necessity to manage events coded with MedDRA prior to statistical analysis: proposal of a strategy with application to a randomized clinical trial, ANRS 099 ALIZE. Contemp Clin Trials 29(2):95-101

Liberati A, Altman DG, Tetzlaff J, Mulrow C, Gøtzsche PC, loannidis JP et al (2009) The PRISMA statement for reporting systematic reviews and metaanalyses of studies that evaluate health care interventions: explanation and elaboration. Ann Intern Med 151(4):W-65-W-94

Lincoln YS, Guba EG (1986) But is it rigorous? Trustworthiness and authenticity in naturalistic evaluation. New directions for program Eval. 1986(30):73-84

Methodology WCCfDS (2011) ATC structure and principles: WHO [cited 2014]. http://www.whocc.no/atc/structure_and_principles/

Milne R, Oliver S (1996) Evidence-based consumer health information: developing teaching in critical appraisal skills. Int I Qual Health Care 8(5):439-445

Mitchell AS, Henry DA, Sanson-Fisher R, O'Connell DL (1988) Patients as a direct source of information on adverse drug reactions. BMJ (Clinical research ed) 297(6653):891-893

Moher D, Liberati A, Tetzlaff J, Altman DG (2009) Preferred reporting items for systematic reviews and meta-analyses: the PRISMA statement. Ann Intern Med 151(4):264-269

Mozzicato P (2009) MedDRA. Pharm Med 23(2):65-75 
Oshikoya KA, Awobusuyi JO (2009) Perceptions of doctors to adverse drug reaction reporting in a teaching hospital in Lagos, Nigeria. BMC Clin Pharmacol 9:14

Sharrar RG, Dieck GS (2013) Monitoring product safety in the postmarketing environment. Ther Adv Drug Saf 4(5):211-219. doi:10.1177/2042098613490780

Shea BJ, Hamel C, Wells GA, Bouter LM, Kristjansson E, Grimshaw J et al (2009) AMSTAR is a reliable and valid measurement tool to assess the methodological quality of systematic reviews. J Clin Epidemiol 62(10):1013-1020

Stillwell SB, Fineout-Overholt E, Melnyk BM, Williamson KM (2010) Evidencebased practice, step by step: asking the clinical question: a key step in evidence-based practice. Am J Nurs 110(3):58-61

Stricker Psaty (2004) Detection, verification, and quantification of adverse drug reactions. BMJ Br Med J 329(7456):44
Thorne S, Kirkham SR, MacDonald-Emes J (1997) Interpretive description: a noncategorical qualitative alternative for developing nursing knowledge. Res Nurs Health 20(2):169-177

Thorne S, Armstrong EA, Harris SR, Hislop TG, Kim-Sing C, Oglov V et al (2009) Patient real-time and 12-month retrospective perceptions of difficult communications in the cancer diagnostic period. Qual Health Res 19(10):1383-1394

van Grootheest K, de Graafde L (2003) Jong-van den Berg LT. Consumer adverse drug reaction reporting: a new step in pharmacovigilance? Drug Saf Int J Med Toxicol Drug Exp 26(4):211-217

WHO (2002) The importance of pharmacovigilance [cited 2014 Feb 10]. http:// apps.who.int/iris/bitstream/10665/42493/1/a75646.pdf?ua=1

\section{Submit your manuscript to a SpringerOpen ${ }^{\circ}$ journal and benefit from:}

- Convenient online submission

- Rigorous peer review

- Immediate publication on acceptance

- Open access: articles freely available online

- High visibility within the field

- Retaining the copyright to your article

Submit your next manuscript at $>$ springeropen.com 